\title{
BMJ Open Safety and efficacy of faecal microbiota transplantation by Anaerobic Cultivated Human Intestinal Microbiome (ACHIM) in patients with systemic sclerosis: study protocol for the randomised controlled phase II ReSScue trial
}

To cite: Hoffmann-Vold A-M, Fretheim HH, Sarna VK, et al. Safety and efficacy of faecal microbiota transplantation by Anaerobic Cultivated Human Intestinal Microbiome (ACHIM) in patients with systemic sclerosis: study protocol for the randomised controlled phase II ReSScue trial. BMJ Open 2021;11:e048541. doi:10.1136/ bmjopen-2020-048541

- Prepublication history and additional online supplemental material for this paper are available online. To view these files, please visit the journal online (http://dx.doi.org/10. 1136/bmjopen-2020-048541).

Received 30 December 2020 Accepted 01 June 2021

Check for updates

(c) Author(s) (or their employer(s)) 2021. Re-use permitted under CC BY-NC. No commercial re-use. See rights and permissions. Published by BMJ.

For numbered affiliations see end of article.

Correspondence to Dr Anna-Maria Hoffmann-Vold; a.m.hoffmann-vold@medisin. uio.no

\section{ABSTRACT}

Introduction In the multisystem inflammatory disorder systemic sclerosis (SSc), gastrointestinal tract (GIT) affliction is highly prevalent. There are no known disease modifying therapies and the negative impact is substantial. Aiming for a new therapeutic principle, and inspired by recent work showing associations between gut microbiota changes and GIT symptoms in SSc, we performed a pilot study on faecal microbiota transplantation (FMT) with the single-donor bacterial culture 'Anaerobic Cultivated Human Intestinal Microbiome (ACHIM)'. Motivated by positive pilot study signals, we designed the ReSScue trial as a phase II multicentre, placebo-controlled, randomised 20week trial to evaluate safety and efficacy on lower GIT symptoms of FMT by ACHIM in SSc.

Methods and analyses We aim to include 70 SSC participants with moderate to severe lower GIT symptoms, defined by the validated patient-reported University of California Los Angeles Scleroderma Clinical Trial Consortium GIT 2.0 2.0 questionnaire. The trial includes three parts. In part A1 (induction phase) lasting from week 0 to week 12, participants will be randomised 1:1 to repeat infusions of $30 \mathrm{~mL} \mathrm{ACHIM}$ or placebo at week 0 and 2 by gastroduodenoscopy. In part A2, which is an 8-week subsequent maintenance phase, all study participants will receive $30 \mathrm{~mL} \mathrm{ACHIM}$ at week 12 and followed until week 20 on continued blind. In part B, which will last until the last participant completes part A2, the participants will be followed through a maximum 16-week extended monitoring period, for longer-term data on safety and intervention effects. Primary endpoint is change from baseline to week 12 in UCLA GIT subscale scores of diarrhoea or bloating, depending on the worst symptom at baseline evaluated separately for each patient. Secondary endpoints are safety measures and changes in UCLA GIT scores (total, diarrhoea and bloating).

\section{Strengths and limitations of this study}

- This study is a randomised clinical trial.

- The feasibility of reproduction is high due to the use of Anaerobic Cultivated Human Intestinal Microbiome.

- Long-term safety data will be available.

- The efficacy cannot be assessed beyond the 12week study period.

Ethics and dissemination This protocol was approved by the Northern Norwegian Committee for Medical Ethics. Study findings will be published.

Trial registration number NCT04300426; Pre-results. Protocol version V.3.1.

\section{INTRODUCTION}

Systemic sclerosis (SSc) is a rare, multiorgan system disorder with a marked negative impact on quality of life and reduced survival, with gastrointestinal tract (GIT) involvement as a major contributor to both. ${ }^{1-5}$ There are no specific therapies available for SSc-related GIT disease, but symptomatic relief can be provided by some agents, including proton pump inhibitors. ${ }^{367}$ Understanding of mechanisms behind SSc-related GIT disease is poor. However, it is known that alterations of the gut microbiota (dysbiosis) exist in SSc and associate with specific GIT symptoms. However, the question of how these alterations affect the pathogenesis of this disease and correlate with SSc symptoms still remains. In terms of GIT symptoms, there is evidence 
that altered abundance of certain genera is associated with a reduction or increase in specific GIT symptoms. ${ }^{8-10}$ This provides a rationale for manipulation of the patient's gut microbiota with the intention of attaining therapeutic effects. $^{8-10}$ Faecal microbiota transplantation (FMT) is currently applied as a therapeutic intervention strategy for targeting of gut bacteria in human diseases. ${ }^{11}$ We hypothesise that FMT is a strategy to be further explored in SSc patients and that intervention by Anaerobic Cultivated Human Intestinal Microbiome (ACHIM) in patients with SSc reduces lower GIT symptoms and GIT disease processes through direct effects on the gut microbiome composition and indirect effects on immune networks. We also hypothesise effects on other disease processes driven by constituents of the SSc gut microbiome. ${ }^{12}$

Evidence supporting the concept of FMT for therapeutic purposes comes mainly from relapsing Clostridioides difficile colitis. Early phase controlled studies indicated improved efficacy of FMT by faeces from random healthy donors compared with standard treatment by antibiotics. ${ }^{13}$ FMT with healthy donor faeces has also shown promise in complex GIT disorders, such as the inflammatory bowel diseases and irritable bowel syndrome (IBS) ${ }^{11}{ }^{14}$ It is also under investigation in many other disorders, including a range of metabolic conditions. ${ }^{11}$ It appears that clinical efficacy is highly dependent on the properties of the donor faeces in most studied diseases, at the same time limiting possibilities for reproducibility. ${ }^{11}$ In general, it appears that FMT is well tolerated, but several safety issues remain unresolved, such as the potential cotransfer of resistant bacteria and viruses. ${ }^{15} 16$ Recently, concerns were raised by a report showing that two immunecompromised patients had contracted drug-resistant E. coli from donor faeces, with lethal outcome in one of them. ${ }^{17}$ These safety issues, as well as donor variation is feasible to overcome by replacing donor faeces with standardised in vitro cultivated bacterial ecosystems. Products of this type are available, such as the ACHIM, which was originally derived from faeces of a single healthy Norwegian donor. ${ }^{14}$ ACHIM is cultivated in vitro under strictly anaerobic conditions, with maintained stability of bacterial species composition. Clinically, it appears that the efficacy of FMT by ACHIM in C. difficile colitis is comparable to that of FMT by fresh random donor feces. ${ }^{18}$ Of notice, and of special interest in times of the ongoing COVID-19 pandemic, ACHIM has due to repeated recultivation using media selecting for bacteria without using any human components been tested to be free of human materials, known viruses and bacterial resistance genes, and has been so for the past two decades. ${ }^{19}$ Work is in progress to develop protocols for regular screening of known pathogens in donor faeces to be applied for FMT. But as the COVID-19 pandemic illustrates, it may not be possible to identify all potential human pathogens in fresh human faeces before it is given to patients. ${ }^{1219}$

It is in general challenging to perform and interpret clinical trials in a rare, complex and heterogeneous disorder like SSc. Additionally, for an FMT trial with donor faeces, there are specific challenges with response evaluation, not only due to marked inter-patient variations in extent and severity of GIT symptoms at baseline, but also to differences in faecal microbiota composition between donors, evaluated by bacterial gene sequencing. ${ }^{9}$ Thus, when we set up the first FMT pilot trial in SSc, we decided to standardise the intervention by using ACHIM. This allowed for (1) repeat, identical FMT across the active intervention group, (2) possibilities for systematic tracking of donor microbiome across the intervention group and importantly (3) possibility for independent trial replications. ${ }^{20}$

In SSc, there are no clinical disease activity indices that include GIT parameters, and no data on defined GIT outcomes from any SSc intervention study. ${ }^{21}$ Thus, for the FMT by ACHIM pilot trial it was not possible to benchmark GIT endpoints against previous intervention studies. ${ }^{20}$ Therefore, we had to develop a primary endpoint, with rationale for it derived from observational studies. We chose the patient-reported outcome measure (PROM) 'University of California Los Angeles Scleroderma Clinical Trial Consortium GIT 2.0' (UCLA GIT) questionnaire. ${ }^{22}$ This PROM was developed for cross-sectional and longitudinal assessment of severity and impact of GIT symptoms in SSc and has been tested in recent clinical trials. It was therefore relevant for the purpose of the pilot trial. ${ }^{22} 23$

The UCLA GIT assesses GIT symptom severity in SSc by 34 items in seven multi-item scales. Five of the scale scores (reflux, bloating, diarrhoea, faecal soilage and constipation) grade severity of upper and lower GI symptom. The two last assess impact of GI symptoms on emotional well-being and social participation. ${ }^{22}$ For the pilot trial it was important that the UCLA GIT was equipped with definitions of minimally clinically important differences (MCIDs) for all scale scores included in the total score, and that the score was validated for the Norwegian language..$^{22} 23$

The results from the pilot indicated that FMT by intestinal ACHIM was safe and induced short-term meaningful clinical effects of on the UCLA lower GIT symptom scale scores 'bloating' and 'diarrhoea'. This provided the rationale for further investigations of FMT by ACHIMs safety and efficacy in patients with lower GIT involvement in SSc. ${ }^{20}$

The pilot trial results indicated that effects of ACHIM waned over time. ${ }^{20}$ This is in line with findings from trials in relapsing C. difficile colitis and IBS where responses to FMT peaked after days, lasted for 8-12 weeks and then tended to decrease. ${ }^{24}$ These findings fit the notion that gut microbiota in many chronic diseases states is set to a state of dysbiosis; and will return to this state some time after cessation of intervention. ${ }^{25}$ Therefore, it appears the rationale to design FMT protocols for chronic diseases with an induction therapy phase aiming for rapid 'normalisation' of the patient's gut microbiota, and a maintenance therapy phase aiming to uphold this 'normalised' state. 


\begin{tabular}{|c|c|c|c|}
\hline $\begin{array}{c}\text { Screening period } \\
\text { (up to 12 weeks) }\end{array}$ & $\begin{array}{c}\text { 12-week } \\
\text { induction phase }\end{array}$ & $\begin{array}{c}\text { 8-week } \\
\text { maintenance phase }\end{array}$ & $\begin{array}{c}\text { maximum } 16 \text { week } \\
\text { monitoring phase }\end{array}$ \\
\hline
\end{tabular}

Double-blind

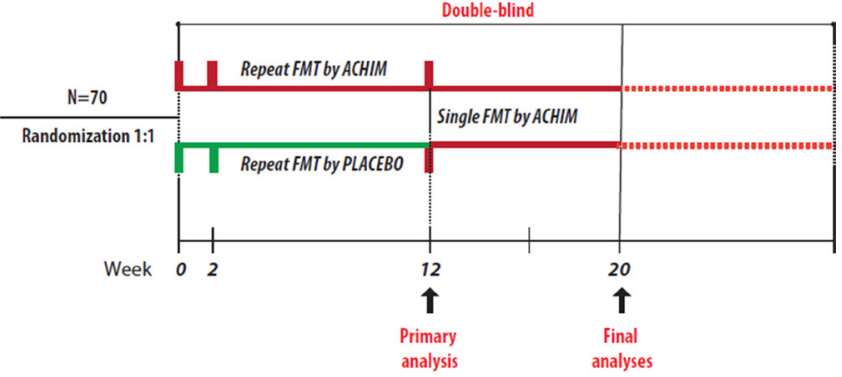

B 20 weeks
\begin{tabular}{|l|l|l|}
\hline Induction & malnten & monitoring \\
\hline
\end{tabular}

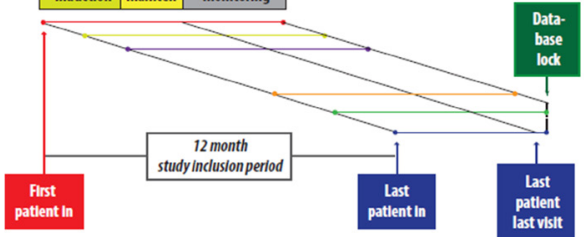

Figure 1 ReSScue study schematic with overall trial design and on participant level $(A)$ overall trial design $(B)$ trial design on participant level. ACHIM, Anaerobic Cultivated Human Intestinal Microbiome; FMT, faecal microbiota transplantation

Ideally, one should assess safety and efficacy of FMT induction and FMT maintenance in SSc in separate controlled trials. But due to the limited number of eligible SSc patients in Norway, this remains challenging. Therefore, we designed ReSScue with two sequential phases. First, a 12-week induction phase (part A1) with repeat intestinal infusions, followed by an 8-week maintenance phase (part A2) with a single intestinal infusion of ACHIM at week 12 in all study participants (figure 1A). Additionally, we plan to follow all participants for a maximum 16 weeks until the last patient completes the 20 weeks (part B) to obtain longer-term data on safety and durability of the effects (figure 1B). ${ }^{26-28}$ Importantly, we maintain the initial induction phase blind for all participants until the end of the study. Study end is defined as the time point when the last study participant has completed the last study visit at the end of the 20-week study period. By keeping the blind, we will be able to provide pilot data indicating if a single ACHIM infusion is sufficient as stand-alone therapy for lower GIT symptoms, or need to be combined with induction by repeat intestinal infusions.

Originally, we designed the ReSScue study with two modes of FMT delivery: (1) FMT by intestinal infusions in the induction phase (A1) and (2) FMT by daily doses of oral ACHIM capsules for the maintenance phase. The rationale for this approach was that oral capsules would be easier to administrate than intestinal infusions and transferable to daily clinical practice. Moreover, based on results from C. difficile colitis studies, we hypothesised that clinical efficacy of per oral capsules would not be inferior to intestinal infusions. ${ }^{29}$ Unfortunately, the production line for oral ACHIM capsules will not be ready in time for the ReSScue trial, but should be available for later studies.
The effects of FMT by ACHIM is presumably mediated through direct effects on the patient's gut microbiome composition, and possibly also through indirect effects on immune networks, with the potential for clinical effects outside the GIT. ${ }^{30} \mathrm{We}$, thus, hypothesise that intervention by ACHIM in SSc patients will result in clinically meaningful improvement of patient reported lower GIT symptoms, compared with placebo, and have effects on other disease processes driven by constituents of the SSc gut microbiome.

\section{METHODS AND ANALYSIS Trial design}

The ReSScue trial is a multicentre Phase II, randomised, double-blind placebo-controlled 20-week study consisting of three parts including $70 \mathrm{SSc}$ participants (figure 1). It is an investigator initiated RCT with the Oslo University Hospital as the sponsor (affiliation No 1). The study consists of a 4-12 week screening period, a 12-week induction phase (part A1), with 1:1 randomised, double-blind parallel group interventions by endoscopic infusions of ACHIM or placebo at weeks 0 and 2, and an 8-week maintenance phase (part A2) where all participants receive one single open label endoscopic infusion of ACHIM at week 12 (figure 1A). Additionally, we will follow all participants for a maximum 16 weeks monitoring period until the last patient completes the study (Part B) to obtain longerterm data on safety and durability of intervention effects (figure 1B). ${ }^{27}{ }^{28}$ We used the Standard Protocol Items: Recommendations for Interventional Trials reporting guidelines. ${ }^{31}$

\section{Study setting}

The study will be performed at four university hospitals in Norway. Participants for the study will be recruited from the entire SSc population in Norway, with study inclusion regulated by the trial eligibility criteria explained below. Participant recruitment, eligibility screening, retrieval of informed consent and data collection will be performed at the four ReSScue study sites. Dietary habits at time of inclusion and at every study visits are recorded.

\section{Objectives}

The primary objective of this trial is to estimate efficacy of intestinal ACHIM infusions compared with placebo infusions on lower GIT symptoms. This is measured by change from baseline to week 12 in UCLA GIT scale scores of diarrhoea or bloating, depending on the worst symptom at screening. The trial is set up with four secondary objectives, including safety objectives focusing on tolerability of FMT by ACHIM and additional GIT measures.

\section{Eligibility criteria}

Detailed eligibility criteria are listed in online supplemental.

The major inclusion criteria are:

- Females and Males with age 18 to 85 years. 
- Fulfilment of the 2013 ACR/EULAR classification criteria for SSc.

- Ability to understand and follow trial procedures including completion of questionnaires regarding PROMs, such as the UCLA GIT score.

- Presence of moderate to severe SSc-related lower GI symptoms at time of inclusion, as defined by above threshold UCLA GIT score values for bloating and/ or diarrhoea.

The major exclusion criteria are:

- End-stage organ disease, including cardiovascular disease, lung disease, pulmonary hypertension, gastrointestinal conditions, renal insufficiency.

- Antibiotic therapy within 3 months of visit 1 .

- Prednisone $>10 \mathrm{mg} /$ day within 4 weeks, and cyclophosphamide or rituximab treatment within 6 months prior to visit 1.

- Unstable background therapy with; mycophenolate mofetil/sodium, methotrexate, azathioprine, tocilizumab, abatacept, leflunomide, tacrolimus, tofacitinib and cyclosporine A (see online supplemental for details).

\section{Interventions}

In part A1, eligible patients will be randomised 1:1 to receive $30 \mathrm{~mL}$ ACHIM or placebo, administered as duodenal infusions by upper endoscopy at weeks 0 and 2. In part A2, all the study participants will receive $30 \mathrm{~mL}$ ACHIM, administered as duodenal infusions by upper endoscopy at week 12 (figure 1). Both study interventions will be provided in dark, sealed tubes ensuring similar appearance of placebo and ACHIM.

\section{Outcomes}

This study is designed with primary endpoint, defined by change from baseline to week 12 in the worst of the two UCLA GIT scale scores 'diarrhoea' or 'bloating' (table 1). ${ }^{22}$ Worst symptom is decided individually for each patient based on the screening visit GIT scale score values. As the two scores are measured on different scales, the diarrhoea score is rescaled into equivalent scale by multiplying it by 1.5 . The subscale with the highest (rescaled) score is defined as the worst symptom. In case of equal scores for bloating and diarrhoea at screening, diarrhoea is chosen as the predominant symptom. If only one of the scores is available at screening, then that is used as the worst symptom.

Secondary endpoints include safety measures as well as 'total and scale scores diarrhoea and bloating' assessed by the UCLA GIT score. Safety and tolerability of FMT will be assessed by adverse event (AE) monitoring, physical examination, vital signs and clinical laboratory testing from baseline to the end of the study at week 20 .

As the scientific rationale for intervention studies in SSc is limited by insufficient understanding of disease mechanisms, we plan ReSScue as a proof-of-concept study. The study is designed with repeated samplings of biospecimens including faeces, urine, blood, salvia and skin samples. This allows for broad range of explorative biomedical endpoints during study parts A1 and A2 and part B, and later development of research studies at molecular level.

The primary and secondary efficacy objectives will be evaluated the end of part A1 at week 12. Secondary safety endpoints and explorative clinical endpoints will be assessed at weeks 12 and 20 and partly 36 .

\section{Participant timeline}

ReSScue is designed with one visit over a maximum 12-week screening period and a 12-week induction phase (part A1) with five visits at week $0,2,4,6$ and 12 (table 2). The maintenance phase (part A2) is an 8 weeks phase with three visits at week 14, 16 and 20 (table 2). Patients completing part A will go over to the monitoring phase (part $\mathrm{B}$ ) with a maximum of additional 16 weeks, or until the last patient reaches 20 weeks of part A2. The phase consists of maximum four visits at week 24, 28, 32 and 36 (figure 1A,B).

\section{Sample size}

Based on power calculations for the primary endpoint 'change in worst symptom from baseline to week 12', we plan to enrol in total approximately 70 adult Norwegian patients with SSc at the four ReSScue sites. Assumptions on the MCIDs and SD of the GIT scale scores diarrhoea and bloating used in the calculation are adopted from Khanna et $a l^{23}$ However, in contrast to the validation study that was based on two assessments of UCLA GIT score, this study will assess UCLA GIT score four times before baseline and five times during the study part A1. Thus, it can be argued that this study has better control over the variance, and the assumption on the SD used is potentially conservative. A sample size of approximately $\mathrm{N}=64$ patients, $\mathrm{n}=32$ patients per ACHIM/placebo arm, is expected to achieve a power of $80 \%$ of detecting a change in mean $(\mathrm{p}<0.05$, two sided) of -0.50 (or higher) if the relating SD would be 0.70 (or lower). Considering a dropout rate of $10 \%$, a total of approximately 70 patients will be enrolled in the study.

\section{Recruitment}

Recruitment of participants has started in September 2020. Study inclusion started 28 September 2020 and will end 30 April 2022.

\section{Assignment of interventions}

Based on previous data on the same patient population, the proportions of the worst lower GIT symptoms in patients with SSc are not equal; $40 \%$ patients having diarrhoea, and $60 \%$ bloating as worst symptom. To ensure equal allocation of different symptoms to each intervention group, a stratified randomisation procedure is implemented. Participants are first grouped into strata according to the worst symptom (diarrhoea/bloating), and then assigned to an intervention according to separate randomisation schedules within each stratum. Worst symptom is decided individually based on the screening visit GIT score values. 
Table 1 Efficacy endpoints in all study parts

\section{Endpoints}

Primary Change from baseline to week 12 in UCLA GIT score items diarrhoea or bloating, depending which was the worst symptom at the baseline evaluated separately for each patient.

Secondary Safety and tolerability assessed by adverse event (AE) monitoring, physical examination and clinical laboratory testing from baseline to the end of the study period Change from baseline to week 12 in total UCLA GIT score Change from baseline to week 12 in UCLA GIT score item diarrhoea Change from baseline to week 12 in UCLA GIT score item bloating

Other outcome measures

Change from baseline to week 12 in Faecal incontinence quality of life scale

Change from baseline to week 12 in UCLA GIT score item reflux

Change from baseline to week 12 in UCLA GIT score item faecal soilage

Change from baseline to week 12 in UCLA GIT score item constipation

Change from baseline to week 12 in UCLA GIT score item emotional well-being

Change from baseline to week 12 in UCLA GIT score item social participation

Change from baseline to week 12 in - Change from baseline to week 12 in HAQ-DI scores

Change from baseline to week 12 in VAS Fatigue scale

Change from baseline to week 12 in Sclerold score

Change from baseline to weeks 2, 6 and 12 in overall faecal microbiome composition measured by 16sRNA based methods

Change from baseline to week 12 in saliva, skin and urine microbiome measured by 16 sRNA based methods

Change from baseline to weeks 2, 6 and 12 in immunoglobulin bound fraction of the overall faecal microbiome

Change from baseline to week 12 in GIT time and contractions evaluated by SmartPill technology along with registration of stool frequency and consistency by Bristol Stool Scale

Change from baseline to weeks 6 and 12 in peripheral blood B cell and T cells (as evaluated by receptor sequencing, proteomics and cellular phenotyping) and content of soluble molecules

Change from baseline to weeks 2 and 12 in the architecture and cellular composition of duodenal biopsy specimens (including characterisation of cellular surface markers, proteomics, metabolomics and immune cell receptor sequencing

Change from baseline to week 12 in skin properties evaluated by elastography and ultrasonographic skin thickness

Change from baseline to week 12 in Health-related quality of life assessed by EQ-5D

Change from week 12 through week 20 in all participants, and up to week 36 in a subset of participants in UCLA GIT score item diarrhoea or bloating, depending which was the worst symptom at the baseline evaluated separately for each patient

Follow changes of UCLA total GIT score from week 12 through week 20 in all participants, and up to week 36 in a subset of participants

Follow changes in mean of HAQ-DI; VAS Fatigue; Sclerold score; and patient reported global assessment from week 12 through week 20 in all participants, and up to week 36 in a subset of participants Assess changes of overall faecal microbiome composition measured by 16sRNA-based methods from week 12 to week 20 in all participants, and up to week 36 in a subset of participants

Assess change of saliva, skin and urine microbiome measured by 16 sRNA based methods from week 12 to week 20 in all participants

Assess change in peripheral blood B cell and T cells and content of soluble molecules from week 12 to week 20 in all participants, and up to week 36 in a subset of participants

Assess changes in upper GIT scores from week 12 to 20 and determine potential associations to the architecture and cellular composition of oesophagus biopsy specimens

Assess change from week 12 to 20 in Health-related Quality of Life assessed by EQ-5D from week 12 to week 20 in all participants, and up to week 36 in a subset of participants

EQ5D, EuroQoL 5L - health-related quality of life; HAQ-DI, 1qHealth Assessment Questionnaire-Disability Index; UCLA GIT, University of California Los Angeles Scleroderma Clinical Trial Consortium Gastrointestinal Tract; VAS, Visual Analogue Scale.

On day 1 , the study participants will be assigned a unique number (randomisation number) in ascending numerical order at the Oslo University Hospital (OUH). The randomisation number encodes the participant's assignment to the two intervention arms in study part A1 according to the randomisation schedule generated prior to the study by statisticians at the OUH study site and entered in the same web-based solution as the electronic case report forms (eCRF). Each participant will be dispensed blinded study intervention, labelled with his/her unique randomisation number, throughout the 12-week study. 


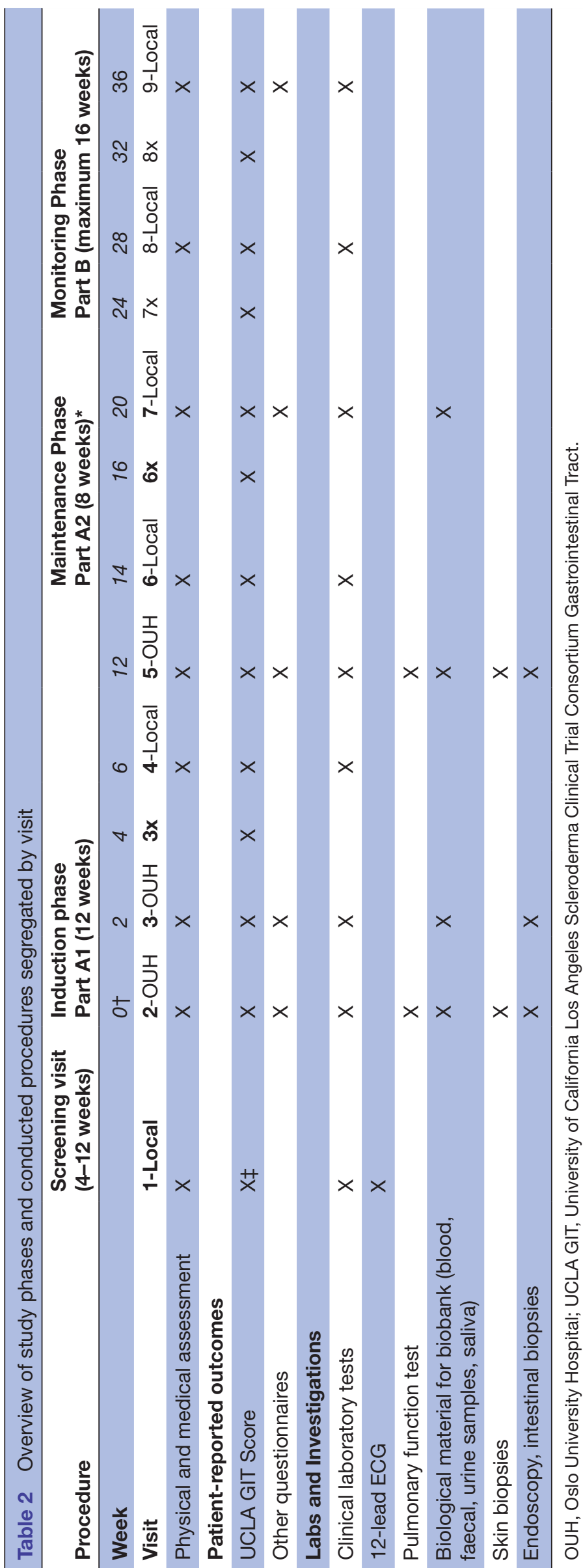

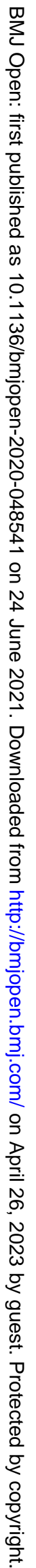


After completion of the part A1, 12-week doubleblinded study, the participant's randomisation number will remain blinded throughout part A2. Unblinding of the randomisation number will not be performed before all the participants have completed the 20 weeks ReSScue trial study period and data have been locked.

\section{Data collection, management}

Data recording will follow the Good Clinical Practice reporting guidelines. Data will be collected with eCRF. For this data recording and handling Viedoc, part of the NorCRIN structure, will be used. NorCRIN is the Norwegian hub of a larger European research network, European Clinical Research Infrastructure Network. Patient specific modules with, that is, EQ-5D already exist, the others will be developed in collaboration with the clinical trial unit (CTU) at OUH. The investigators will have access to the final trial dataset without any limitations. Data management is provided from the CTU at OUH.

\section{Statistical methods}

Continuous primary endpoint 'UCLA GIT scores of subscales diarrhoea or bloating' is assessed four times during the screening period and five times during the study period A1 (at week 0, 2, 4, 6 and 12), and will be analysed using a linear mixed model accounting for the correlations between repeated measurements within each participant by random intercept and slope. The intervention group slope, stratification factor (worst symptom) and intervention*time-interaction will be treated as fixed effects in the model. In addition, the model will be adjusted for the baseline value of the patient specific worst symptom.

The continuous secondary endpoints 'total UCLA GIT score', 'UCLA GIT scale score diarrhoea' and 'UCLA GIT scale score bloating' will be analysed exploiting the repeated measurements at nine time points (four during the screening period and five at weeks $0,2,4,6$ and 12) by using a linear mixed model with the intervention group, stratification factor and intervention*time-interaction as fixed effects and a random intercept and slope.

For safety, summary tables will be created for all safety information. All site-reported AEs will also be grouped by seriousness, primary relationship (intervention, unrelated and unknown) and timing of onset (preintervention and postintervention).

\section{Data monitoring}

An independent data monitoring committee (DMC) is established, following good standard DMC guidelines which includes insights to blinded and unblinded data. The DMC contains a SSc expert, a gastroenterologist with FMT expertise and a statistician. Safety concerns will be discussed in the DMC which meets on a regular basis.

The intensity of AEs will be rated by the investigators as mild (easily tolerated), moderate (enough discomfort to cause interference with usual activity) or severe (incapacitating or causing inability to work or to perform usual activities). AEs of gastrointestinal perforation and laryngospasm due to gastro-duodenoscopy are of special interest, due to previous occurrence in the pilot trial. Patients will be observed at our dedicated clinical trial department after each gastroduodenoscopy for these possible AEs and assessed accordingly if symptoms appear.

\section{Patient and public involvement statement}

Patient representatives from the Norwegian Rheumatology Foundation have been involved in the study protocol development, obtaining funding and will be involved in the conduction of the trial.

\section{ETHICS AND DISSEMINATION}

The trial is being conducted in compliance with the protocol, the ethical principles laid down in the Declaration of Helsinki and in accordance with the International Conference on Harmonisation Tripartite Guideline for Good Clinical Practice. All patients will provide written informed consent prior to trial entry. The study has been approved by the Regional Committee of Health and Medical Research Ethics in Northern Norway (No. 2016/1529) and the Norwegian Medical Agency (Statens legemiddelverk) (20/02597-21). Study findings will be shared at conferences and as published manuscripts; and made available to interested participants. No medical writers will be used.

\section{Author affiliations}

${ }^{1}$ Department of Rheumatology, Oslo University Hospital, Oslo, Norway

${ }^{2}$ Faculty of medicine, University of Oslo, Oslo, Norway

${ }^{3}$ Department of gastroenterology, Oslo University Hospital, Oslo, Norway ${ }^{4}$ Department of Rheumatology, University Hospital Zurich, Zurich, Switzerland

${ }^{5}$ Division of Rheumatology, University of Michigan, Ann Arbor, Michigan, USA

${ }^{6}$ Division of Rheumatology, David Geffen School of Medicine, Los Angeles,

California, USA

${ }^{7}$ Department of Rheumatology, St Olavs Hospital Universitetssykehuset i Trondheim, Trondheim, Norway

${ }^{8}$ Department of Rheumatology, University of Bergen, Bergen, Hordaland, Norway

${ }^{9}$ Department of Rheumatology, University Hospital of North Norway, Tromso, Troms, Norway

${ }^{10}$ Oslo Centre for Biostatistics \& Epidemiology, Oslo University Hospital, Oslo, Norway

Contributors A-MH-V: conceived the presented idea and developed the theory; conception and design of the study, conduction and has drafted the manuscript and has given final approval of the version to be published. HHF: has been involved in the planning, conduction and reporting of the work described in the article. VKS: has been involved in the planning, conduction and reporting of the work described in the article. IB: has been involved in the planning, conduction and reporting of the work described in the article. MNC: has been involved in the planning, conduction and reporting of the work described in the article. OD: has been involved in the planning including conception and design of the study, has been involved in drafting the manuscript and has given final approval of the version to be published. DK: has been involved in the planning including conception and design of the study, has been involved in drafting the manuscript and has given final approval of the version to be published. ERV: has been involved in the planning including conception and design of the study, has been involved in drafting the manuscript and has given final approval of the version to be published. ØMi: has been involved in the planning, conduction and reporting of the work described in the article. HD: has been involved in the planning, conduction and reporting of the work described in the article. $A D$ : has been involved in the planning, conduction and reporting of the work described in the article. A-KH: has been involved in the planning, conduction 
and reporting of the work described in the article. GB: has been involved in the planning, conduction and reporting of the work described in the article. MP: conception and design of the study, has been involved in the planning, conduction and reporting of the work described in the article. 10: conception and design of the study, has been involved in the planning, conduction and reporting of the work described in the article. ØMo: conceived the presented idea and developed the theory; has been involved in the planning, conduction and reporting of the work described in the article.

Funding This study is funded by Clinical Therapy Research in the Specialist Health Services (KLINBEFORSK) Norway.

Disclaimer The funder has no role in the collection, management, analysis, and interpretation of data; writing of the report; and the decision to submit the report for publication, and does not have any ultimate authority over any of these activities.

Competing interests A-MH-V: Actelion, ARXX, Bayer, Boehringer Ingelheim, Medscape, Merck Sharp \& Dohme, Lilly and Roche. OD: Abbvie, Acceleron Pharma, Amgen, AnaMar, Bayer, Boehringer Ingelheim, Catenion, Drug Development International, CSL Behring, ChemomAb, GSK, Horizon (Curzion) Pharmaceuticals, Inventiva, Italfarmaco, iQvia, Lilly, Medac, Medscape, Mitsubishi Tanabe Pharma, MSD, Novartis, Pfizer, Roche, Sanofi, Serodapharm, Target Bio Science and UCB in the area of potential treatments of scleroderma and its complications. In addition, OD has a patent mir-29 for the treatment of systemic sclerosis issued (US8247389, EP2331143). DK: Abbvie, Actelion/Janssen, Acceleron Pharma, Amgen, Bayer, Boehringer Ingelheim, CSL Behring, GSK, Horizon Pharmaceuticals, Mitsubishi Tanabe Pharma, Pfizer, Roche, Sanofi, United Therapeutics. DK is chief medical officer of Eicos Sciences. ERV: Disclosures unrelated to the manuscript: Grants (Corbus, Forbius, Boehringer Ingelheim); Consulting fees (Boehringer Ingelheim). $\emptyset$ Mi: Son of owner of ACHIM.

Patient consent for publication Not required.

Provenance and peer review Not commissioned; externally peer reviewed.

Supplemental material This content has been supplied by the author(s). It has not been vetted by BMJ Publishing Group Limited (BMJ) and may not have been peer-reviewed. Any opinions or recommendations discussed are solely those of the author(s) and are not endorsed by BMJ. BMJ disclaims all liability and responsibility arising from any reliance placed on the content. Where the content includes any translated material, BMJ does not warrant the accuracy and reliability of the translations (including but not limited to local regulations, clinical guidelines, terminology, drug names and drug dosages), and is not responsible for any error and/or omissions arising from translation and adaptation or otherwise.

Open access This is an open access article distributed in accordance with the Creative Commons Attribution Non Commercial (CC BY-NC 4.0) license, which permits others to distribute, remix, adapt, build upon this work non-commercially, and license their derivative works on different terms, provided the original work is properly cited, appropriate credit is given, any changes made indicated, and the use is non-commercial. See: http://creativecommons.org/licenses/by-nc/4.0/.

ORCID iDs

Anna-Maria Hoffmann-Vold http://orcid.org/0000-0001-6467-7422

Dinesh Khanna http://orcid.org/0000-0003-1412-4453

\section{REFERENCES}

1 Denton CP, Khanna D. Systemic sclerosis. The Lancet 2017;390:1685-99.

2 Frantz C, Avouac J, Distler O, et al. Impaired quality of life in systemic sclerosis and patient perception of the disease: a large international survey. Semin Arthritis Rheum 2016;46:115-23.

3 Hoffmann-Vold A-M, Volkmann ER. Gastrointestinal involvement in systemic sclerosis: effects on morbidity and mortality and new therapeutic approaches. Journal of Scleroderma and Related Disorders 2021;6:37-43

4 Fretheim H, Halse A-K, Seip M, et al. Multidimensional tracking of phenotypes and organ involvement in a complete nationwide systemic sclerosis cohort. Rheumatology 2020;59:2920-9.

5 Hughes M, Heal C, Siegert E, et al. Significant weight loss in systemic sclerosis: a study from the EULAR scleroderma trials and research (EUSTAR) database. Ann Rheum Dis 2020;79:1123-5.

6 Shreiner AB, Murray C, Denton C, et al. Gastrointestinal manifestations of systemic sclerosis. J Scleroderma Relat Disord 2016;1:247-56.
7 Hansi N, Thoua N, Carulli M. Consensus best practice pathway of the UK scleroderma Study group: gastrointestinal manifestations of systemic sclerosis. Clin Exp Rheumatol 2014;32(6 Suppl 86):S-21421.

8 Volkmann ER, Chang YL, Barroso N. Systemic sclerosis is associated with a unique colonic microbial consortium. Arthritis Rheumatol 2016;68:1483-92.

9 Volkmann ER, Hoffmann-Vold A-M, Chang Y-L, et al. Systemic sclerosis is associated with specific alterations in gastrointestinal microbiota in two independent cohorts. BMJ Open Gastroenterol 2017;4:e000134.

10 Volkmann ER, Hoffmann-Vold A-M. Gastrointestinal tract microbiota modifications in systemic sclerosis. Eur J Rheumatol 2020;7:228-36.

11 Ooijevaar RE, Terveer EM, Verspaget HW, et al. Clinical application and potential of fecal microbiota transplantation. Annu Rev Med 2019;70:335-51.

12 Hoffmann-Vold A-M, Fretheim $\mathrm{H}$, Didriksen $\mathrm{H}$, et al. The potential of fecal microbiota transplantation in systemic sclerosis. Expert Rev Clin Immunol 2020;16:117-8.

13 van Nood E, Vrieze A, Nieuwdorp M, et al. Duodenal infusion of donor feces for recurrent Clostridium difficile. $N$ Engl J Med 2013;368:407-15.

14 Benno P, Norin E, Midtvedt T, et al. Therapeutic potential of an anaerobic cultured human intestinal microbiota, ACHIM, for treatment of IBS. Best Pract Res Clin Gastroenterol 2019;4041:101607.

15 Rosenbaum JT. Just another crappy commentary: the future of fecal microbiota transplantation. Expert Rev Clin Immunol 2019;15:987-9.

16 Verbeke F, Janssens Y, Wynendaele E, et al. Faecal microbiota transplantation: a regulatory hurdle? BMC Gastroenterol 2017;17:128.

17 DeFilipp Z, Bloom PP, Torres Soto M, et al. Drug-Resistant E. coli Bacteremia Transmitted by Fecal Microbiota Transplant. N Engl J Med 2019;381:2043-50.

18 Juul FE, Garborg K, Bretthauer M, et al. Fecal microbiota transplantation for primary Clostridium difficile infection. N Engl J Med 2018;378:2535-6

19 laniro G, Mullish BH, Kelly CR, et al. Screening of faecal microbiota transplant donors during the COVID-19 outbreak: suggestions for urgent updates from an international expert panel. Lancet Gastroenterol Hepatol 2020;5:430-2.

20 Fretheim $\mathrm{H}$, Chung BK, Didriksen $\mathrm{H}$, et al. Fecal microbiota transplantation in systemic sclerosis: a double-blind, placebocontrolled randomized pilot trial. PLoS One 2020;15:e0232739.

21 Khanna D, Nagaraja V, Gladue $\mathrm{H}$, et al. Measuring response in the gastrointestinal tract in systemic sclerosis. Curr Opin Rheumatol 2013;25:700-6.

22 Khanna D, Hays RD, Maranian P, et al. Reliability and validity of the University of California, Los Angeles scleroderma clinical trial Consortium gastrointestinal tract instrument. Arthritis Rheum 2009;61:1257-63.

23 Khanna D, Furst DE, Maranian P, et al. Minimally important differences of the UCLA scleroderma clinical trial Consortium gastrointestinal tract instrument. J Rheumatol 2011;38:1920-4.

24 Johnsen PH, Hilpüsch F, Cavanagh JP, et al. Faecal microbiota transplantation versus placebo for moderate-to-severe irritable bowel syndrome: a double-blind, randomised, placebo-controlled, parallel-group, single-centre trial. Lancet Gastroenterol Hepatol 2018;3:17-24.

25 Clemente JC, Manasson J, Scher JU. The role of the gut microbiome in systemic inflammatory disease. BMJ 2018;360:j5145.

26 Distler O, Brown KK, Distler JHW, et al. Design of a randomised, placebo-controlled clinical trial of nintedanib in patients with systemic sclerosis-associated interstitial lung disease (SENSCIS ${ }^{\mathrm{TM}}$ ). Clin Exp Rheumatol 2017;35(Suppl 106):75-81.

27 Distler O, Highland KB, Gahlemann M, et al. Nintedanib for systemic sclerosis-associated interstitial lung disease. $N$ Engl J Med 2019;380:2518-28.

28 Flaherty KR, Wells AU, Cottin V, et al. Nintedanib in progressive fibrosing interstitial lung diseases. N Engl J Med 2019;381:1718-27.

29 Kao D, Roach B, Silva M, et al. Effect of oral Capsule- vs Colonoscopy-Delivered fecal microbiota transplantation on recurrent Clostridium difficile infection: a randomized clinical trial. JAMA 2017;318:1985.

30 Palm NW, de Zoete MR, Flavell RA. Immune-microbiota interactions in health and disease. Clin Immunol 2015;159:122-7.

31 Chan A-W, Tetzlaff JM, Gøtzsche PC, et al. SPIRIT 2013 explanation and elaboration: guidance for protocols of clinical trials. BMJ 2013;346:e7586-e86. 